\title{
Driving forces for the adsorption of enzymes at the water/silica interface studied by total internal reflection fluorescence spectroscopy and optical reflectometry
}

\author{
C. Czeslik ${ }^{\mathrm{a}, *}$, G. Jackler ${ }^{\mathrm{a}}$ and C. Royer ${ }^{\mathrm{b}}$ \\ ${ }^{a}$ Universität Dortmund, Physikalische Chemie I, Otto-Hahn-Str. 6, D-44221 Dortmund, Germany \\ ${ }^{\mathrm{b}}$ Université de Montpellier 1, Centre de Biochimie Structurale, F-36060 Montpellier, France
}

\begin{abstract}
Total internal reflection fluorescence (TIRF) spectroscopy has been used to study conformational changes of hen egg white lysozyme induced by interaction with the water/quartz interface. TIRF spectra have been measured over a large temperature range and are compared to the corresponding solution spectra. It has been found that the wavelength of maximum fluorescence intensity of adsorbed lysozyme is red-shifted by about $7 \mathrm{~nm}$ relative to that of dissolved lysozyme in the temperature range of about $20-60^{\circ} \mathrm{C}$. This observation indicates that lysozyme is partially unfolding when it is adsorbing on quartz. Using optical reflectometry a drastic temperature-induced increase of the degree of adsorption of lysozyme and staphylococcal nuclease (SNase) on silicon wafers has been measured which suggests that the corresponding adsorption processes are endothermic and thus entropically driven. The major contribution to this entropy gain will probably originate from conformational changes at lower temperatures. The experimental results indicate that proteins with a smaller Gibbs energy of unfolding have a higher tendency for adsorption. Above the temperatures of unfolding of the proteins in solution, the dehydration of hydrophobic residues, which are exposed to water in the thermally unfolded state, are the most likely driving force for the adsorption of lysozyme and SNase on silicon oxide.
\end{abstract}

\section{Introduction}

The adsorption of protein molecules at aqueous/solid interfaces is an interesting phenomenon that is also relevant for medical diagnostics or protein analysis and purification [1,2]. A knowledge of the driving forces underlying the protein adsorption processes is important for both the optimization of protein adsorption applications and the prevention of a protein coverage on materials, e.g., medical implants. Driving forces responsible for the adsorption of proteins at aqueous/solid interfaces, as discussed in the literature, include enthalpic and entropic contributions to the standard Gibbs energy of adsorption, such as Coulomb and van der Waals interactions, protein conformational changes, the release of counterions and the dehydration of hydrophobic surface areas [3-5].

Although the variation of temperature can reveal a more detailed insight into the mechanism of protein adsorption, only a relatively small number of temperature-dependent studies exists in the literature [6-8]. For example, the comparison of the temperature-induced unfolding of a protein in the dissolved and the adsorbed state provides information about the strength of protein-surface interactions. As has been found in a recent fluorescence spectroscopy study, the temperature, enthalpy and entropy

\footnotetext{
*Corresponding author. Tel.: +49 231755 3903; Fax: +49 231755 3901; E-mail: claus@ steak.chemie.uni-dortmund.de.
} 
of unfolding of lysozyme adsorbed on colloidal silica particles are strongly decreased as compared to dissolved lysozyme, which can be explained by a surface-induced destabilization of lysozyme [7]. In the present study, the adsorption of enzymes on a plane silica surface is investigated as a function of temperature in order to analyze the contribution of protein conformational changes to the driving forces for adsorption. By increasing the temperature, the Gibbs energy of unfolding of a protein can be decreased continuously, thereby promoting adsorption-induced conformational changes which will contribute to the driving forces. A combination of two experimental techniques, total internal reflection fluorescence (TIRF) spectroscopy and optical reflectometry will be applied in this study. Temperature-dependent optical reflectometric experiments will reveal the effect of temperature on the degree of protein adsorption, which will tell us, if the adsorption process is driven by an increase in entropy or a decrease in enthalpy. TIRF spectra of adsorbed lysozyme as a function of temperature will show the extent of conformational modifications upon adsorption, when they are compared with the corresponding solution spectra.

The globular enzymes hen egg white lysozyme and staphylococcal nuclease (SNase) are used as model proteins in this study. Both proteins are of comparable size with 129 and 149 amino acid residues, respectively. At $\mathrm{pH}=7$, they carry a net positive charge owing to their isoelectric points of 11.1 (lysozyme) and 9.5 (SNase). However, they differ in their stability against thermal unfolding. The temperature of unfolding of lysozyme is reported in the literature to lie between 70 and $78^{\circ} \mathrm{C}$ and the Gibbs energy of unfolding is $65 \mathrm{~kJ} \mathrm{~mol}^{-1}[9]$. Thus, the stability of folded lysozyme molecules can be varied over a large temperature range. In contrast, SNase is unfolding at $49-54^{\circ} \mathrm{C}$ and has a lower Gibbs energy of unfolding of $24 \mathrm{~kJ} \mathrm{~mol}^{-1}$, which allows one to investigate the effect of protein conformation (folded vs. unfolded) on the degree of adsorption at moderate temperatures [10]. Although lysozyme is often classified as a "hard" protein which largely preserves its native structure at an interface, we will show in this study that there are conformational changes when lysozyme adsorbs on silica and that the associated entropy increase is probably the major driving force for adsorption on a hydrophilic surface like silicon oxide.

\section{Experimental section}

Salt-free hen egg white lysozyme was purchased from Roche Diagnostics (Mannheim, Germany) and was used without further purification. Staphylococcal nuclease (SNase) was obtained as described previously [10]. The proteins had a purity of $>99 \%$ which was checked by gel electrophoresis (SDSPAGE). Protein stock solutions were prepared using $10 \mathrm{mM}$ phosphate buffer $(\mathrm{pH}=7.0)$. Final protein concentrations were determined from UV-absorptions at $280 \mathrm{~nm}$ using extinction coefficients of $\varepsilon=2.65 \mathrm{mg}^{-1} \mathrm{ml} \mathrm{cm}^{-1}$ for lysozyme [11] and $\varepsilon=0.93 \mathrm{mg}^{-1} \mathrm{ml} \mathrm{cm}^{-1}$ for SNase [12]. The Si wafers used for optical reflectivity experiments were kindly donated by Wacker Siltronic (Burghausen, Germany). They were cut to pieces of approximately $1.5 \mathrm{~cm} \times 2 \mathrm{~cm}$ and heated to $1000^{\circ} \mathrm{C}$ for about $3 \mathrm{~h}$. An oxide layer of roughly $100 \mathrm{~nm}$ thickness is formed by this treatment, which is necessary in order to detect a protein adsorbate by optical reflectometry. The quartz prism used for TIRF experiments was purchased from Aachener Quarz-Glas Technologie Heinrich (Aachen, Germany). It had a size of $47 \mathrm{~mm}$ $\times 15 \mathrm{~mm} \times 15 \mathrm{~mm}$ with an angle of $70^{\circ}$ between a small side and the largest side. The substrates were cleaned with a mixture of $60 \mathrm{ml} \mathrm{H}_{2} \mathrm{SO}_{4}(96 \%), 30 \mathrm{ml} \mathrm{H}_{2} \mathrm{O}_{2}(30 \%)$ and $10 \mathrm{ml}$ water for about $15 \mathrm{~min}$ at $90^{\circ} \mathrm{C}$. Contact angles of $<14^{\circ}$ were measured for water on the Si wafers and a contact angle of $27^{\circ}$ was found for water on the quartz prism.

Optical reflectivity measurements of the adsorption of lysozyme and SNase at the silica/water interface have been carried out by reflecting the light of a He-Ne laser $(632.8 \mathrm{~nm}, 3 \mathrm{~mW})$ at the surface of a 
thermally oxidized Si wafer, which was positioned in a heatable sample cell filled with protein solution. The incident laser beam made an angle of $70^{\circ}$ to the Si wafer normal and was polarized using a rotatable Glan-Taylor polarizer. The thickness of the oxide layer of a new Si wafer was determined by filling the sample cell with pure buffer and polarizing the incident light with an angle of $45^{\circ}$ to the plane of reflection. The reflected light was polarized successively with angles of $0^{\circ}$ and $90^{\circ}$ using a second rotatable Glan-Taylor polarizer. From the ratio of the corresponding light intensities, $S=I_{\|} / I_{\perp}$, the oxide layer thickness was calculated as described in the literature assuming a 3-layer structure model, $\mathrm{Si} / \mathrm{SiO}_{2} /$ water [13]. For adsorption experiments, the second polarizer was replaced by a polarizing beam splitter cube in order to detect $I_{\|}$and $I_{\perp}$ simultaneously. Following the analysis procedure presented in the literature the relative change of $S$ is proportional to the adsorbed mass of lysozyme per surface area, $\Gamma[14]$ :

$$
\frac{S-S_{0}}{S_{0}}=A \Gamma,
$$

where the index 0 indicates the signal without protein. The adsorption sensitivity $A$ was calculated on the basis of a 4-layer structure model, $\mathrm{Si}_{/} / \mathrm{SiO}_{2} /$ adsorbate/solution using the matrix method of Abelès [13]. Prior to each measurement, the temperature of the sample cell has been adjusted, a small volume of protein stock solution has been added to the buffer in the sample cell, and the sample was equilibrated for $30 \mathrm{~min}$.

The TIRF sample cell consisted of the quartz prism described above, which was separated from a quartz plate by a silicone rubber gasket of $4 \mathrm{~mm}$ thickness. The interior of the cell was rinsed with protein solution using two syringe needles drilled through the rubber gasket. Temperature of the TIRF sample cell was adjusted using a water jacket. The sample cell was positioned in the sample chamber of a K2 fluorescence spectrometer from ISS (Champaign, Illinois, USA) which was operated in photon counting mode. By internal reflection of a monochromatic light beam of $295 \mathrm{~nm}$ at the quartz prism/protein solution interface, an evanescent wave into the solution was generated, by which the Trp residues of protein molecules adjacent to the quartz prism surface were excited [15]. With typical values of $143 \mathrm{~nm}$ for the penetration depth of the evanescent wave, $0.1 \mathrm{mg} \mathrm{ml}^{-1}$ for the protein solution concentration and $2 \mathrm{mg} \mathrm{m}^{-2}$ for the protein surface coverage, one can calculate that more than $99 \%$ of all excited protein molecules are adsorbed on the quartz surface. Fluorescence spectra of dissolved lysozyme were measured using a standard cuvette. All fluorescence spectra were recorded over the wavelength range $315-450 \mathrm{~nm}$. They were normalized to the incident light intensity and were corrected for the wavelength-dependent response of the detection system. Background spectra were recorded with pure buffer and subtracted from the observed fluorescence spectra. After the first filling of the TIRF cell with protein solution, the sample has been equilibrated for $30 \mathrm{~min}$. After a fluorescence spectrum was recorded, the temperature of the sample cell was increased by $5^{\circ} \mathrm{C}$, the sample cell was rinsed with fresh protein solution, and the sample was equilibrated for $15 \mathrm{~min}$ before taking a new spectrum.

Experimental errors are given in the figure captions, they are derived from at least two independent measurements.

\section{Results and discussion}

In Fig. 1, intrinsic fluorescence spectra of lysozyme are shown for dissolved lysozyme at low and high temperature and for lysozyme, which is adsorbed on a quartz surface at room temperature. In the case 
of dissolved lysozyme, the maximum of the fluorescence band is shifted by about $9 \mathrm{~nm}$ to higher wavelengths, when the temperature is increased from 23 to $84^{\circ} \mathrm{C}$. This shift can be explained by the thermal unfolding of the native lysozyme structure. In the unfolded state, the Trp residues of lysozyme are exposed to water molecules, which lowers the energy difference between the ground and the electronically excited state of the fluorophores due to solvent relaxation [16]. A red-shift of the fluorescence peak of about $7 \mathrm{~nm}$ is observed (Fig. 1), when lysozyme adsorbs on a quartz surface. A similar effect has also been reported by Buijs and Hlady [17]. An adsorption-induced red-shift of the lysozyme fluorescence band suggests that the lysozyme molecules will partially unfold when interacting with a quartz surface. The intrinsic fluorescence of native lysozyme is mainly $(>80 \%)$ emitted from the Trp residues 62 and 108, which are located in the active site [18]. Trp 108 is less exposed to water and emits at shorter wavelengths. Thus, upon adsorption, it is likely that the environment of the more buried Trp 108 residue is changing and becoming more accessible for water molecules. However, it must be noted that unfolding of lysozyme increases the fluorescence contribution of the other four Trp residues significantly [18].

In Fig. 2, the temperature dependence of the wavelength of maximum fluorescence intensity is shown for dissolved and adsorbed lysozyme. With increasing temperature, dissolved lysozyme shows a slight blue-shift of this wavelength in the temperature range of $20-50^{\circ} \mathrm{C}$, which might be related to a decreasing fractional intensity of the Trp 62 residue. At higher temperatures, lysozyme forms an intermediate with intact secondary structure and collapsed or highly flexible tertiary structure, which is characterized

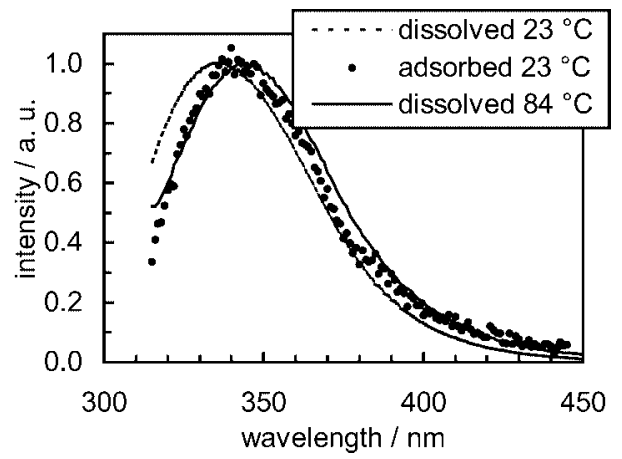

Fig. 1. Fluorescence spectra of hen egg white lysozyme $\left(\mathrm{pH}=7.0\right.$, protein solution concentration $0.08 \mathrm{mg} \mathrm{ml}^{-1}$, excitation wavelength $295 \mathrm{~nm}$ ). The spectrum of adsorbed lysozyme has been recorded using a TIRF sample cell with lysozyme molecules adsorbed on a quartz surface.

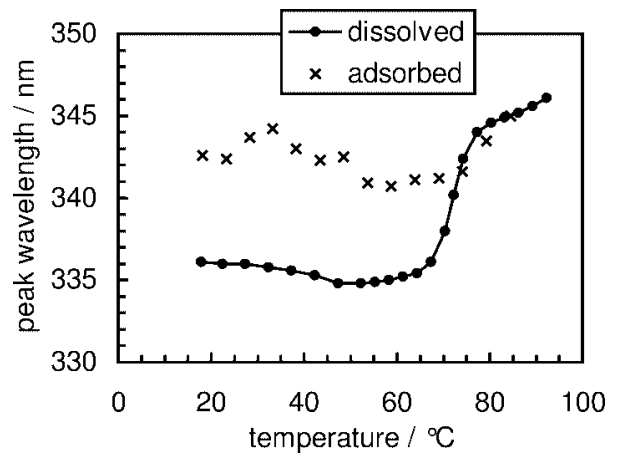

Fig. 2. Wavelength of maximum fluorescence intensity of lysozyme as a function of temperature, see Fig. 1 for details. Maximum experimental errors are $\pm 0.5 \mathrm{~nm}$ for dissolved and $\pm 2 \mathrm{~nm}$ for adsorbed lysozyme. 


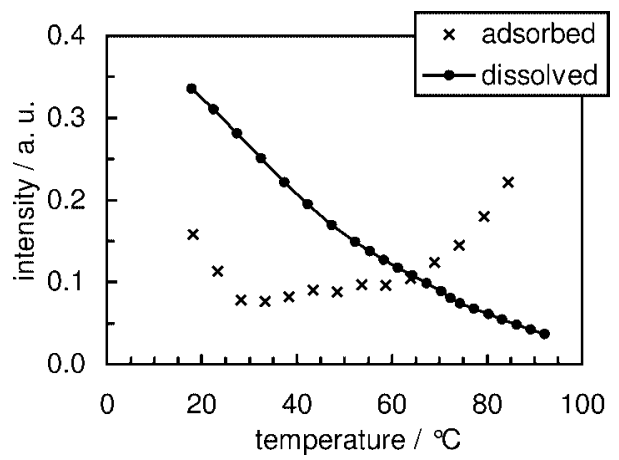

Fig. 3. Total fluorescence intensity of lysozyme in the wavelength range $320-450 \mathrm{~nm}$ as a function of temperature, see Fig. 1 for details. Maximum experimental errors are $\pm 5 \%$ for dissolved and $\pm 15 \%$ for adsorbed lysozyme.

by an enhanced H/D exchange rate of labile hydrogen atoms [19]. Above $50^{\circ} \mathrm{C}$, the peak wavelength of the fluorescence band of dissolved lysozyme is increasing (Fig. 2), consistent with an enhanced water accessibility of the Trp residues in the intermediate state. Within the experimental error, adsorbed lysozyme is characterized by maximum fluorescence intensity at $342-343 \mathrm{~nm}$ at temperatures below $70^{\circ} \mathrm{C}$, which suggests that at these temperatures significant conformational changes occur in lysozyme when adsorbing on a plane quartz surface. Above $75^{\circ} \mathrm{C}$, where dissolved lysozyme is thermally unfolded, the fluorescence spectra of dissolved and adsorbed lysozyme largely coincide.

It is interesting to analyze the total fluorescence intensity emitted by lysozyme in the wavelength range of 320-450 nm (Fig. 3). Whereas this intensity is decreasing in the case of dissolved lysozyme over the whole temperature region studied, increasing intensity with increasing temperature is observed from adsorbed lysozyme above about $30^{\circ} \mathrm{C}$. For dissolved lysozyme, the formation of an intermediate and the thermal unfolding are reflected in very small changes of the temperature dependence of the total fluorescence intensity at about 50 and $70^{\circ} \mathrm{C}$. For adsorbed lysozyme, the unusual temperature-dependence of the total fluorescence intensity may be explained by two opposing effects: a temperature-induced decrease of the fluorescence intensity due to a decreasing average fluorescence lifetime, and a temperature-induced increase of the fluorescence intensity due to an increasing amount of adsorbed lysozyme molecules. As has been observed before, the average fluorescence lifetime of lysozyme adsorbed on colloidal silica particles is reduced at elevated temperatures [7].

In order to quantify the degree of lysozyme adsorption on a silica surface as a function of temperature, optical reflectometric measurements have been performed. In Fig. 4, the results of these measurements are shown. For comparison, data are also given for the less stable protein SNase. Similar protein solution concentrations have been chosen as that used in the TIRF experiments. As can be seen in Fig. 4, the degree of lysozyme and SNase adsorption at the silica/water interface is strongly affected by temperature. Upon unfolding of the proteins in solution, the degree of adsorption is strongly increasing. A small temperature-induced enhancement of adsorption is already observed for both proteins below their temperatures of unfolding in solution, which suggests that the adsorption processes are endothermic and thus entropically driven. The most probable entropic driving force for the folded proteins will be a conformational change, which increases the reorientational mobility of protein segments. This conclusion is supported by the TIRF study of lysozyme described above, in which a partial unfolding of lysozyme has been found upon adsorption at the silica/water interface in the temperature range of $20-60^{\circ} \mathrm{C}$. With increasing temperature, the Gibbs energy of unfolding of a protein is lowered thereby promoting the 


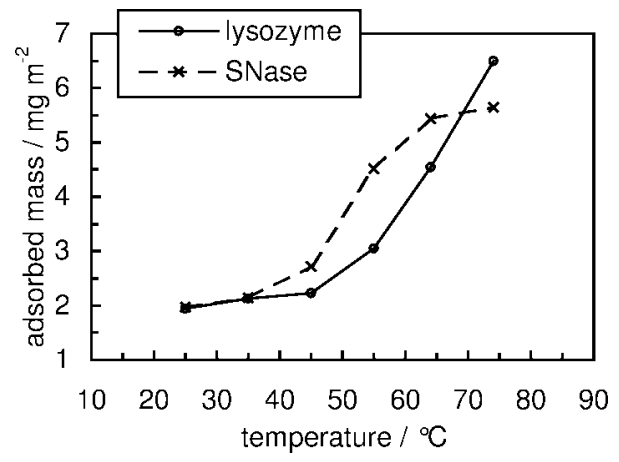

Fig. 4. Adsorbed protein mass per surface area as a function of temperature. The data have been recorded using optical reflectometry with oxidized silicon wafers as substrates. The protein solutions had a pH-value of 7.0 and a concentration of $0.09 \mathrm{mg} \mathrm{ml}^{-1}$. The data have an experimental error of about $\pm 5 \%$.

readiness for an adsorption-induced conformational change which results in an increased number of adsorbed protein molecules.

A conformational modification of the protein molecules cannot be the major driving force for adsorption at higher temperatures where the dissolved protein molecules are thermally unfolded. When a protein is unfolded, apolar amino acid residues are exposed to water. These residues will then be partially dehydrated by interacting with the silica surface or other protein molecules, which are already adsorbed. This hydrophobic dehydration leads to a favorable entropy increase due to the increasing reorientational mobility of water molecules. From an inspection of Fig. 4, it appears that a hydrophobic dehydration is a very strong driving force for protein adsorption, because lysozyme and SNase molecules show a larger degree of adsorption when they are unfolded in solution. The drastic increase of lysozyme adsorption at the silica/water interface with increasing temperature, as found with optical reflectometry (Fig. 4), can explain the increase of the total fluorescence intensity emitted by adsorbed lysozyme molecules at elevated temperatures (Fig. 3).

\section{Conclusions}

In this study, the driving forces responsible for the adsorption of lysozyme and SNase at the silica/water interface have been discussed. It has been found by optical reflectometry that the degree of adsorption of both proteins is increasing with increasing temperature, which suggests that the adsorption processes are governed by an increase in entropy. At lower temperatures, where the dissolved protein molecules are mainly folded, conformational modifications appear to dominate this entropy gain, whereas at higher temperatures, where the protein molecules are mainly unfolded, a hydrophobic dehydration will be the most important contribution to the increase in entropy. Using TIRF spectroscopy conformational modifications of lysozyme upon adsorption at the silica/water interface have been found over a large temperature range, which supports these conclusions.

\section{Acknowledgements}

This work was supported in part by the Deutsche Forschungsgemeinschaft. 


\section{References}

[1] T.A. Horbett and J.L. Brash, ed., Proteins at interfaces II, ACS Symposium Series 602, Washington, DC, 1995.

[2] S.R. Weinberger, T.S. Morris and M. Pawlak, Recent trends in protein biochip technology, Pharmacogenomics 1 (2000), $395-416$.

[3] V.P. Zhdanov and B. Kasemo, Van der Waals interaction during protein adsorption on a solid covered by a thin film, Langmuir 17 (2001), 5407-5409.

[4] W. Norde, Driving forces for protein adsorption at solid surfaces, Macromol. Symp. 103 (1996), 5-18.

[5] C.M. Roth and A.M. Lenhoff, Electrostatic and van der Waals contributions to protein adsorption: computation of equilibrium constants, Langmuir 9 (1993), 962-972.

[6] G. Jackler, R. Steitz and C. Czeslik, Effect of temperature on the adsorption of lysozyme at the silica/water interface studied by optical and neutron reflectometry, Langmuir, in press.

[7] C. Czeslik and R. Winter, Effect of temperature on the conformation of lysozyme adsorbed to silica particles, Phys. Chem. Chem. Phys. 3 (2001), 235-239.

[8] R.J. Green, I. Hopkinson and R.A.L. Jones, Unfolding and intermolecular association in globular proteins adsorbed at interfaces, Langmuir 15 (1999), 5102-5110.

[9] T.E. Creighton, Proteins, W.H. Freeman and Company, New York, 1993.

[10] G. Panick, G.J.A. Vidugiris, R. Malessa, G. Rapp, R. Winter and C.A. Royer, Exploring the temperature-pressure phase diagram of staphylococcal nuclease, Biochemistry 38 (1999), 4157-4164.

[11] R.F. Steiner, Structural transitions of lysozyme, Biochim. Biophys. Acta 79 (1964), 51-63.

[12] M. Yang, D. Liu and D.W. Bolen, The peculiar nature of the guanidine hydrochloride-induced two-state denaturation of staphylococcal nuclease: a calorimetric study, Biochemistry 38 (1999), 11216-11222.

[13] W.N. Hansen, Electric fields produced by the propagation of plane coherent electromagnetic radiation in a stratified medium, J. Opt. Soc. Am. 58 (1968), 380-390.

[14] J.C. Dijt, M.A. Cohen Stuart and G.J. Fleer, Reflectometry as a tool for adsorption studies, Adv. Colloid Interface Sci. 50 (1994), 79-101.

[15] V. Hlady, R.A. van Wagenen and J.D. Andrade, Total internal reflection intrinsic fluorescence (TIRIF) spectroscopy applied to protein adsorption, in: Surface and Interfacial Aspects of Biomedical Polymers, Vol. 2, J.D. Andrade, ed., Plenum Press, New York, 1985, pp. 81-119.

[16] J.R. Lakowicz, Principles of Fluorescence Spectroscopy, Kluwer Academic, New York, 1999.

[17] J. Buijs and V. Hlady, Adsorption kinetics, conformation, and mobility of the growth hormone and lysozyme on solid surfaces, studied with TIRF, J. Colloid Interface Sci. 190 (1997), 171-181.

[18] T. Imoto, L.S. Forster, J.A. Rupley and F. Tanaka, Fluorescence of lysozyme: emissions from tryptophan residues 62 and 108 and energy migration, Proc. Natl. Acad. Sci. USA 69 (1971), 1151-1155.

[19] I.H.M. van Stokkum, H. Linsdell, J.M. Hadden, P.I. Haris, D. Chapman and M. Bloemendal, Temperature-induced changes in protein structures studied by Fourier transform infrared spectroscopy and global analysis, Biochemistry 34 (1995), 10 508-10 518. 


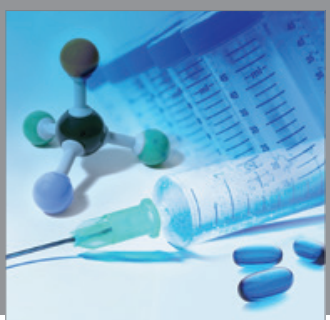

International Journal of

Medicinal Chemistry

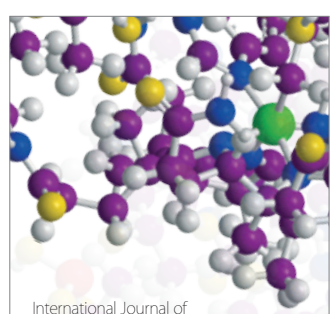

Carbohydrate Chemistry

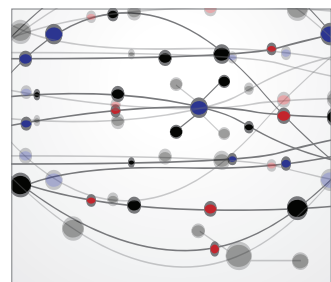

The Scientific World Journal
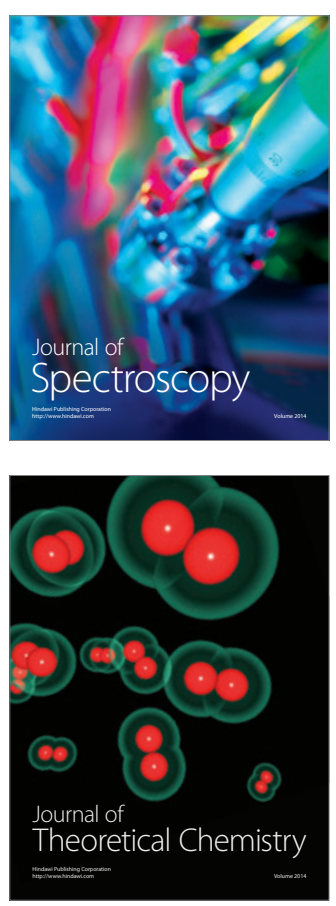
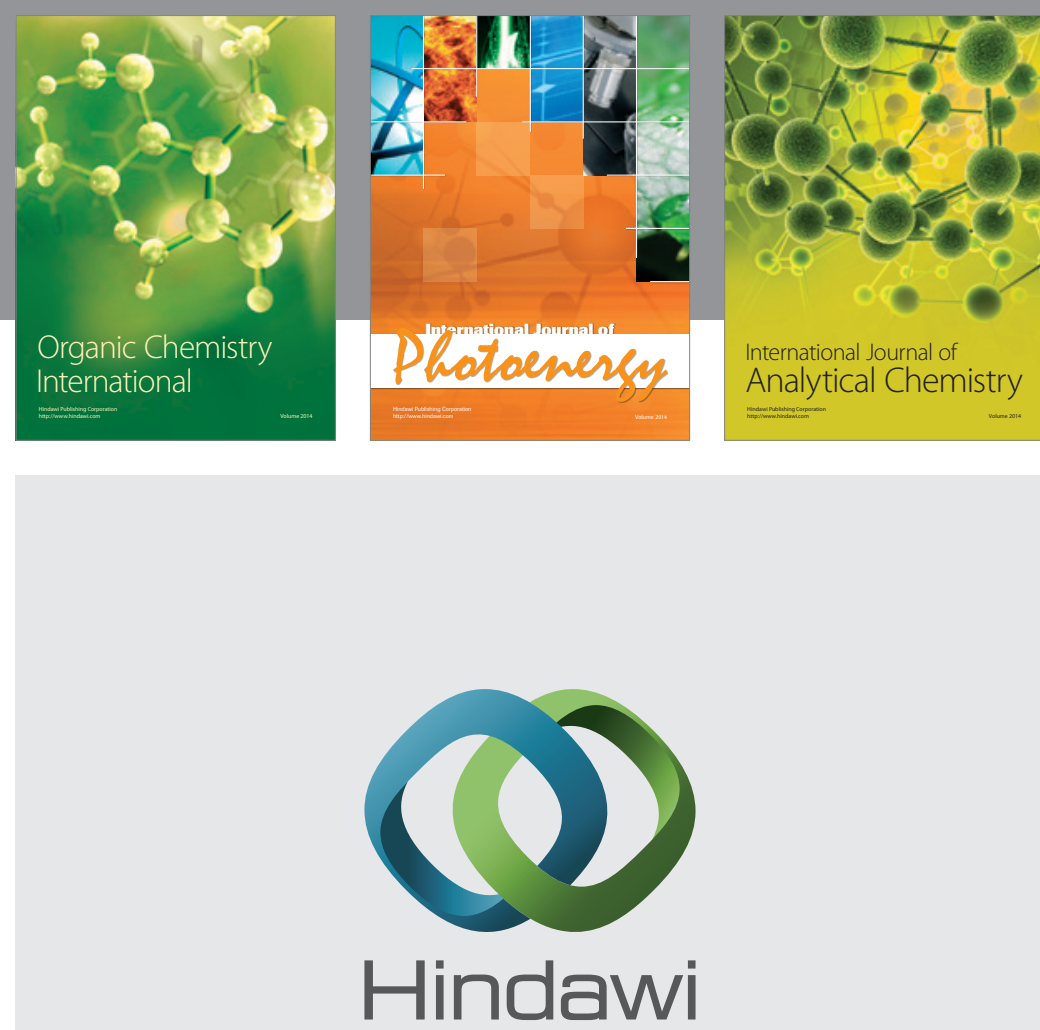

Submit your manuscripts at

http://www.hindawi.com
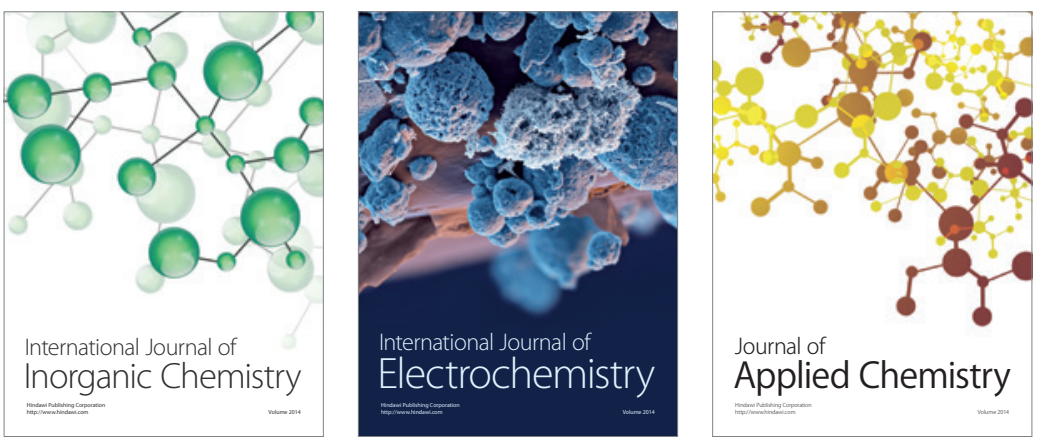

Journal of

Applied Chemistry
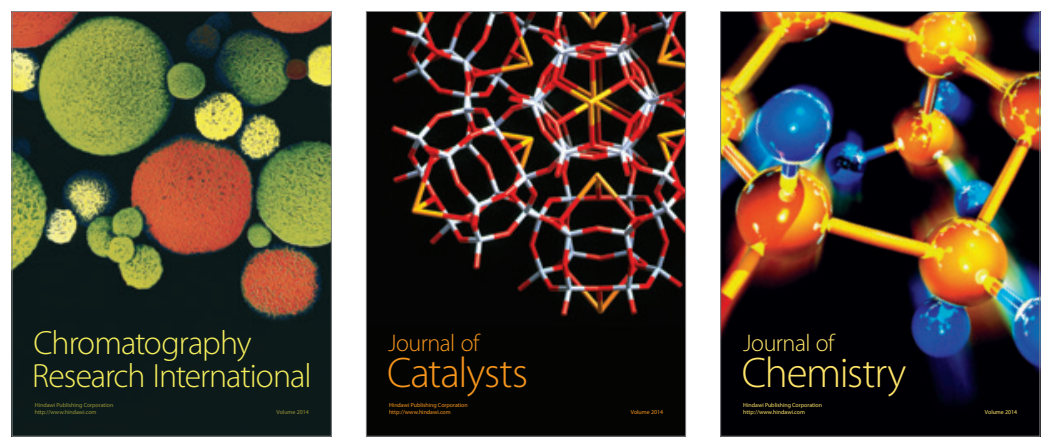
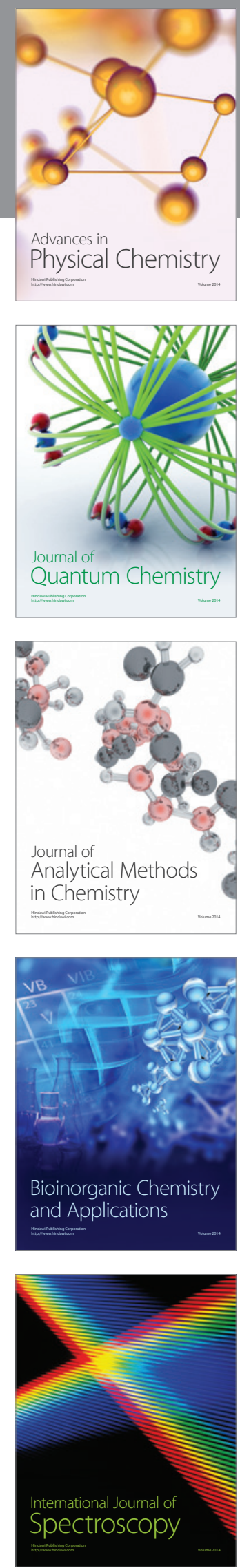